\title{
Concrete structure monitoring with distributed long-gauge optical fiber sensor
}

\author{
Yongsheng Tang \\ School of Urban Rail Transportation, Soochow University, Jixue Road 8\#, Xiangcheng District, \\ Suzhou 215137 , China \\ E-mail: ystang@suda.edu.cn
}

\begin{abstract}
Keywords: long-gauge optical fiber sensor; distributed strain; BOTDA; concrete crack; structural health monitoring.

Abstract: The distributed optical fiber sensing technology, Brillouin Optical Time Domain Analysis (BOTDA), is considered as an effective method for concrete structure monitoring due to its excellent strain sensing performance. However, its sensing accuracy is greatly decreased around the cracks by the influence of spatial resolution. In this paper a new type of optical fiber sensor is proposed based on BOTDA, namely long-gauge sensor, to improve the strain sensing performance especially around concrete cracks. The measurement principle of BOTDA was explained as well as the long-gauge sensor structure and performance. Finally some experiments were implemented to verify the actual sensing performance. From the results it can be concluded that the proposed method can be applied to accurately measure the strain distribution even under the case of large cracks. Considering some other sensing advantages, such as long-term sensing stability, the proposed method presents broad application propects especially in long-term SHM.
\end{abstract}

\section{Introduction}

The concrete structure is the main type of infrastructures due to its low cost especially. However, the concrete crack often develops during the service time, influencing the structure durability or safety. Due to its random distribution, it is not easy to be monitored and identified with the traditional sensing methods. Based on the Brillouin scattering a new optical fiber sensing technique was proposed that strain distribution along the optical fiber (OF) can be obtained at one time even the fiber is up to $20 \mathrm{~km}$, making it possible to identify the random concrete cracks [1]. Since then, many developments have been obtained not only about the application [2, 3] but also about the industrial sensing system from Brillouin Optical Time Domain Reflectometer (BOTDR) to Brillouin Optical Time Domain Analysis (BOTDA) [4, 5]. The strain sensing accuracy has been improved greatly up to $10 \mu \varepsilon$, while the spatial resolution is up to $0.1 \mathrm{~m}$. However, the strain sensing accuracy around the concrete crack will still be greatly influenced by the spatial resolution even it is only $0.1 \mathrm{~m}$, because the complicated strain distribution within the spatial resolution will decrease the strain measurement accuracy greatly [6].

In this paper, a new type of the OF sensor based on BOTDA, namely long-gauge sensor, is proposed to improve the strain sensing performance. Within the sensing gauge the strain distributes uniformly along the OF to ensure the strain measurement accuracy even around the crack. The measurement principle of BOTDA is illustrated at first. Then the improved distributed optical sensor is proposed using the long-gauge structure as well as the sensing performance. Lastly some experiments of a concrete beam are implemented to verify the actual sensing performance.

\section{BOTDA based distributed optical fiber sensing principle}

The BOTDA technique is based on the stimulated Brillouin back scattering, and two laser sources. One is a pulse laser (pump laser) source and the other is a continuous laser source, introduced into the $\mathrm{OF}$ from different ends of the fiber. When the frequency difference between the two lasers is equal to the Brillouin frequency shift, the back Brillouin scattering will be stimulated, and energy transfer will be generated between the two laser. The center frequency of the Brillouin back scattring will move at 
the location where the strain change happens. Thus, the continuous strain measurement along the OF can be implemented by measuring the Brillouin frequency shift. The relationship $v_{B}$ between the Brillouin frequency shift and strain $\varepsilon$ is expressed as Eq. 1.

$$
\mathrm{v}_{B}\left(T_{0}, \varepsilon\right)=C_{\varepsilon}\left(\varepsilon-\varepsilon_{0}\right)+v_{B 0}\left(T_{0}, \varepsilon_{0}\right)
$$

where $C_{\varepsilon}$ is the strain coefficients, while $T_{0}$ and $\varepsilon_{0}$ are the strain and temperature that correspond to a reference Brillouin frequency $v_{B 0}$.

Due to the existence of pulse width, the received back Brillouin scattering does not mean the information for some point of the OF but a small zone. The length of the zone is named as the spatial resolution, calculated as Eq. 2.

$$
\mathrm{S}=v \tau / 2
$$

where $v$ is the light speed in the $\mathrm{OF}$, and $\tau$ is the pulse width. The value of the spatial resolution is needed to be decreased to increase the strain sensing accuracy especially when the strain distribution is complicated. From Eq. 2 the pulse with is only parameter to be decreased. However, there will be some problems, such as poorer signal-to-noise ratios [7].

\section{BOTDA based distributed long-gauge optical fiber sensor}

\section{Long-gauge sensor design}

As shown in Fig. 1, the OF is wrapped with many segments of plastic tube at first, the length of which equals the sensing gauge length. Then, the fiber is braided around the wrapped OF. During this process, the distance between the plastic tube segments should be fixed as the same as the designed bonding length, about $2 \mathrm{~cm} \sim 3 \mathrm{~cm}$. After that some pre-strain should be implemented upon the OF, the amount of which is determined by the actual needs. Finally the epoxy resin is applied to make the long-gauge sensor formed and keep the pre-strain. The distributed long-gauge OF sensor is well prepared with a diameter not larger than $1 \mathrm{~mm}$. Meanwhile the number of the sensing gauges can be also designed as the actual needs.

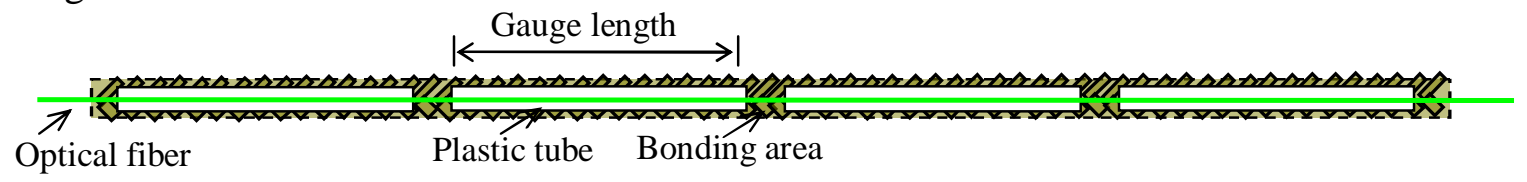

Fig. 1. Distributed long-gauge OF sensor.

\section{Sensing performance}

In Fig. 2, the OFs were bonded on the steel plate surface to investigate the sensing performance around crack. Different gauge length, namely $5 \mathrm{~cm}, 10 \mathrm{~cm}, 15 \mathrm{~cm}, 20 \mathrm{~cm}$ and $30 \mathrm{~cm}$, was set in the tension tests for comparison. These OFs were applied with different strain by changing the crack width. The epoxy resin was selected as the bonding material due to its excellent property.

\section{Gauge length: $5 \mathrm{~cm}, 10 \mathrm{~cm}, 15 \mathrm{~cm}, 20 \mathrm{~cm}, 30 \mathrm{~cm}$}

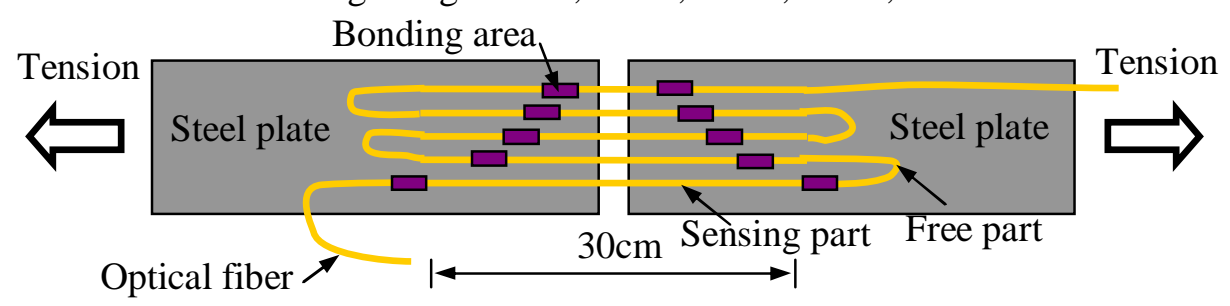

Fig. 2. Experimental setup.

The typical strain distribution is as shown in Fig. 3, from which the sensing parts can be recognized from the free segments easily except the part with $5 \mathrm{~cm}$ gauge length. Actually the strain along the OF is the largest for the $5 \mathrm{~cm}$ gauge length; however, the BOTDA system has obviously failed to measure the strain as the gauge length is smaller than the due to the spatial resolution, $10 \mathrm{~cm}$. 
For the other 4 gauge length, the results present some obvious trend. The larger strain change will be obtained when the gauge length is smaller. In other words, increasing the gauge length will decrease the strain sensitivity to some extent. However, the number of the points within the sensing part shows an inverse trend for extracting the strain average. From the statistics, the higher accuracy will be obtained with the larger sample number. In consideration of the two factors above, the integrated performance should be assessed.

The crack width $w$ was calculated with Eq. 3 to further assess the sensing performance of the proposed distributed OF sensor. In Eq. 3, $\varepsilon$ is the average strain of the gauge length, while $l$ is the gauge length. The results in Fig. 4 have already verified the excellent sensing perfromance as the calculated crack width is close to the actual value with a error only about $0.02 \mathrm{~mm}$.

$$
\omega=\varepsilon l
$$

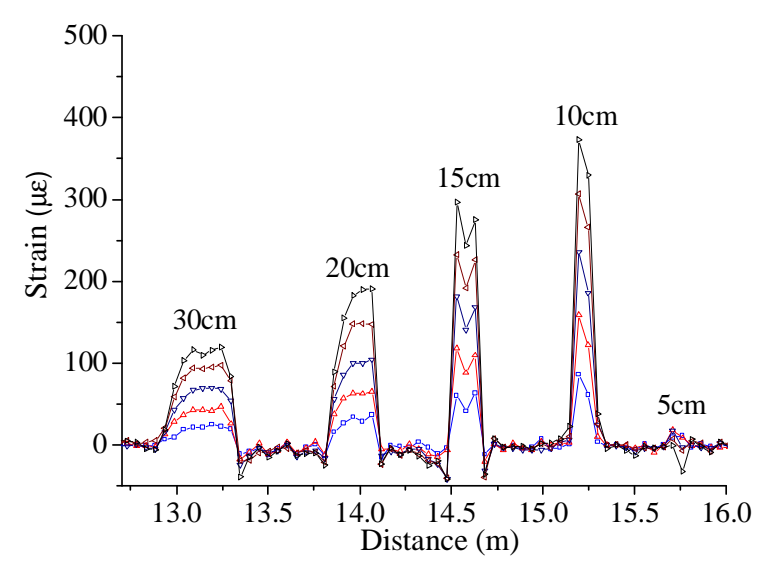

Fig. 3. Typical strain results.

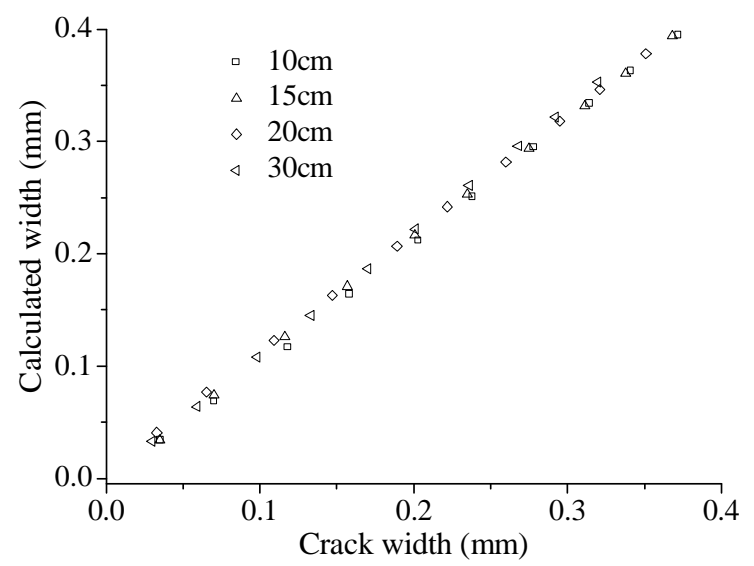

Fig. 4. Crack width results.

\section{Verification with a concrete beam}

\section{Experiment setup}

A reinforced concrete $(\mathrm{RC})$ beam was prepared to implement the verification of the proposed sensor with a rectangle section with a width of $120 \mathrm{~mm}$ and s depth of $240 \mathrm{~mm}$. The beam was simply supported with a span length of $2700 \mathrm{~mm}$ as shown in Fig. 5. Meanwhile 18 long-gauge sensors with a gauge length of $150 \mathrm{~mm}$ in total were installed on the down surface of the beam. The static loads were implemented at the location of $1 / 3$ span step by step until some distinct concrete cracks developed. After some large cracks happening, the beam was reloaded to verified the sensing performance for some special situation, such as earthquake.

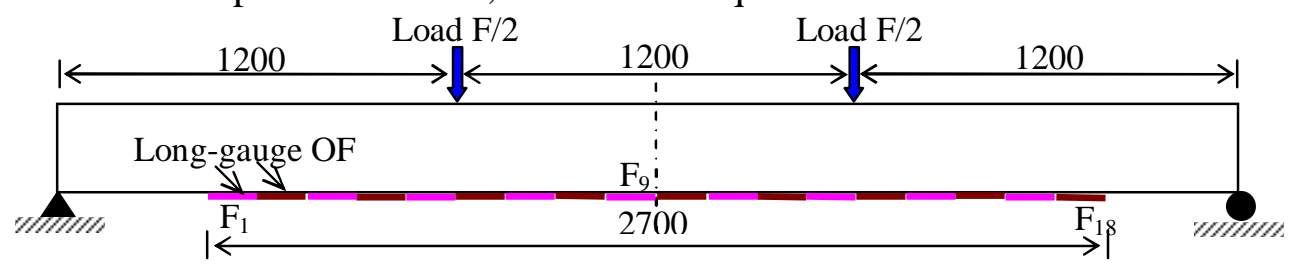

Fig. 5. Experimental setup and sensor installation (Unit: $\mathrm{mm}$ ).

\section{Results and analysis}

The results of strain distribution are shown in Fig. 6, verofying the improvement strain sensing performance especially when concrete cracks develop. In Fig. 6(a), when some cracks happen at the load of $7 \mathrm{kN}$, there are several peaks along the strain distribution compared with the results of the other load case, where the crack is developing within the monitored gauge length. While the peaks are more obvious in Fig. 6(b) as the craks have already developed greatly. Therefore the strain distribution can be accurately obtained with the proposed distributed long-gauge OF sensors even under the case of large cracks. 


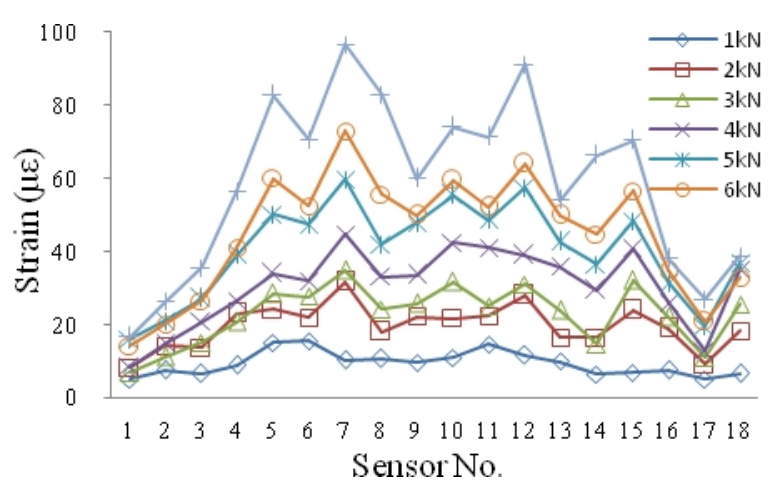

(a)

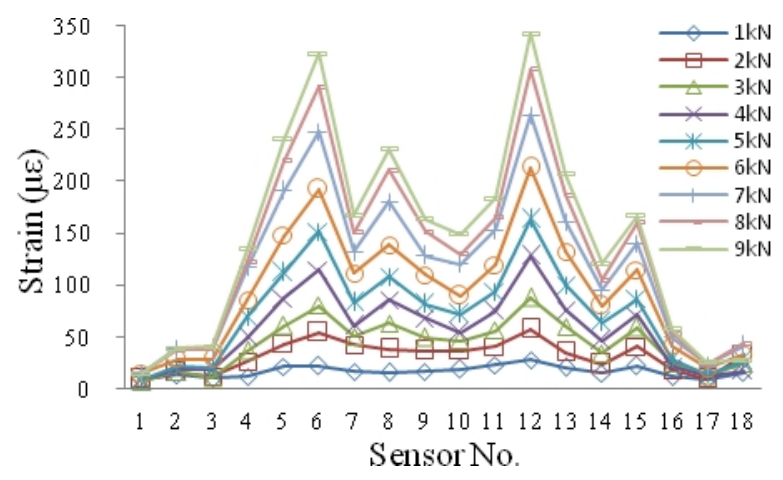

(b)

Fig. 6. Strain distribution at the case of: (a) cracks happening and (b) reloading after large cracks.

\section{Conclusions}

In this paper, a new type of long-gauge sensor OF sensor based on BOTDA is proposed to improve the strain sensing performance especially around concrete cracks. The measurement principle of BOTDA is illustrated and then the long-gauge sensor. Lastly some experiments are implemented to verify the actual sensing performance. Therefore, the conclusion can be drawn that the proposed method can be applied to accurately measure the strain distribution even under the case of large cracks. Combined with the advantages of fiber optic sensing, such as long-term sensing stability, the proposed method presents broad application propects especially in long-term SHM.

\section{Acknowledgements}

This paper is financially supported by University Natural Science Foundation of Jiangsu Province (14KJB580009), National Natural Science Foundation of China (51508364), Natural Science Foundation of Jiangsu Province (BK20150333) and Soochow University Foundation (SDY2014A10).

\section{References}

[1] T. Horiguchi, T. Kurashima and M. Tateda: Tensile Strain Dependence of Brillouin Frequency Shift in Silica Optical Fibers, IEEE Photon. Technol. Lett., 1 (1989), p.107-108.

[2] Z.S. Wu, B. Xu, K. Hayashi and A. Machida: Distributed Optic Fiber Sensing for a Full-scale PC Girder Strengthened with Prestressed PBO Sheets, Engineering Structures, 28 (2006), p.1049-1059.

[3] Z.S. Wu, B. Xu, T. Takahashi and T. Harada: Performance of a BOTDR Optical Fibre Sensing Technique for Crack Detection in Concrete Structures, Journal of Structure and Infrastructure Engineering, 4 (2008), p.311-323.

[4] A.W. Brown, B.G. Colpitts and K. Brown: Dark-Pulse Brillouin Optical Time-Domain Sensor with 20-mm Spatial Resolution, Journal of lightwave technology, 25 (2007), p.381-386.

[5] W.H. Li, X.Y. Bao, Y. Li and L. Chen: Differential Pulse-width Pair BOTDA for High Spatial Resolution Sensing, OPTICS EXPRESS, 16 (2008), p.21616-61625.

[6] H. Zhang and Z.S. Wu: Performance Evaluation of BOTDR-based Distributed Fiber Optic Sensors for Crack Monitoring, Structural Health Monitoring, 7 (2008), p.143-156.

[7] K. Kishida and C.H. Li: Pulse pre-pump-BOTDA technology for new generation of distributed strain measuring system, Proc. Structural Health Monitoring and Intelligent Infrastructure, (2006), p.471-477. 